\title{
Hysteresis Margin and Load Balancing for Handover in Heterogeneous Network
}

\author{
Ranada Prasad Ray and Lun Tang
}

\begin{abstract}
Long term evolution (LTE) heterogeneous network can represent improvement of cell coverage and network capacity by adopting advanced physical layer techniques. In heterogeneous network, one of the major challenges is the handover decision between different eNBs. The utilization of the picocells results to use more network resources and handover procedure. In this paper, we investigate the hysteresis margin and load balancing problem in a 3GPP LTE heterogeneous network. Firstly, we consider A3 event, Hysteresis Margin (HM) and Cell Individual Offset (CIO) objectives are not fixed for every cells. Then we analyze the complexity of the problem and propose a practical algorithm which calculate adaptive hysteresis margin and load balancing in heterogeneous network. The results show that our algorithm can lead to significantly better performances, such as redundant handovers reduction and improvement of the network performance.
\end{abstract}

Index Terms-LTE heterogeneous network, hysteresis margin, cell individual offset, load balancing.

\section{INTRODUCTION}

Heterogeneous networks (HetNets) correspond to a scalable hierarchical cellular network model that is deploying to improve efficiency and increase indoor coverage. Two-tier HetNet comprises conventional MeNBs in the first tier overlaid with the second tier short range, low power and low complexity base stations (corresponding to picocells or femtocells) in Fig. 1 [1]. Because of the smaller coverage area using same licensed frequency band can be efficiently reused multiple times within the second-tier elements of a HetNet, thus improving the capacity and the spectral efficiency per unit area of the network. In HetNet picocells are usually deployed to eliminate coverage holes and improve the capacity of the network. The coverage area of picocells usually varies between $(40-75 \mathrm{~m})$ [2]. 3GPP LTE networks can achieve high spectrum efficiency due to the usage of Multi-Input Multi-Output (MIMO) antenna and orthogonal frequency division multiple (OFDM) technology. However, the network performance is still influenced by several factors, among which different cells and load imbalance.

In mobile communication system cellular structure is the main arrangement. User equipment (UE) often moves through

Manuscript received February 5, 2015; revised June 12, 2015. This work was supported in part by Key Lab of Mobile Communication Technology, Department of Communication and Information at Chongqing University of Posts and Telecommunications, Chongqing, 400065, China.

Ranada Prasad Ray is with the Communication and Information Department, Chongqing University of Posts and Telecommunications, China (e-mail: ranu_ete@yahoo.com).

Lun Tang is with the Chongqing University of Posts and Telecommunications, Chongqing, China (e-mail: tangi@cqupt.edu.cn). different base stations. When UE move to new base stations, call connection will be reconnected to new base stations. This process is called handover. In the same communication networks, the proceeded handoff is called horizontal handover. In heterogeneous communication networks the proceeded handoff is called vertical handover [3]. The strength of signal will be more susceptible to shadowing effect, and be likely to cause handoff between the two base stations back and forth constantly, which is known as the ping-pong effect. By using hysteresis margin value or cell individual offset values the pingpong effect can be significantly reduced. Mobility Load Balancing (MLB) aims to cope with the unequal traffic load between cells. Although the two functions operate independently with each other, there is a close correlation between them, as they both choose adjusting handover parameters as optimization actions.

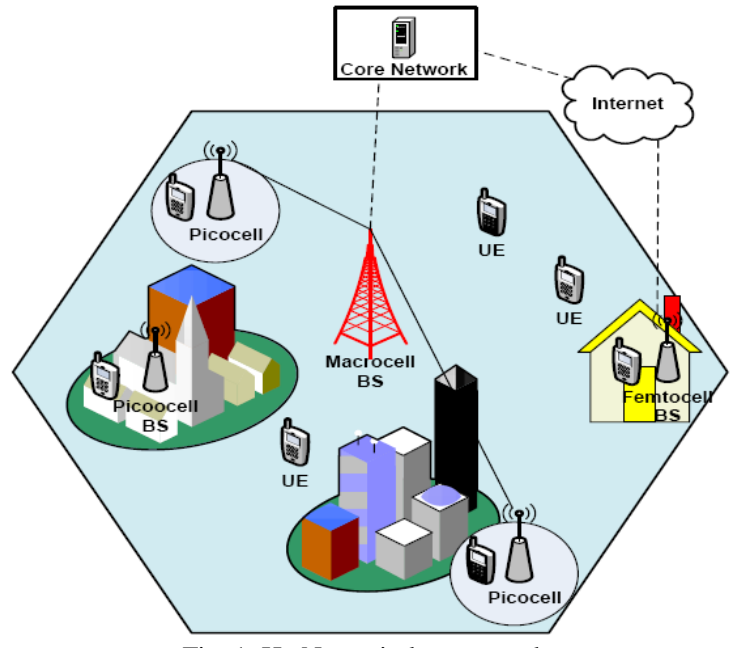

Fig. 1. HetNets wireless network.

The rest of this paper is organized as follows: In Section II the system model and some definitions are proposed. Section III explains the proposed schemes. Section IV evaluates its performances via system level simulations. The paper is concluded in Section V.

\section{SYSTEM MODEL}

We assume that serving cell1 has heavy load but target cell2 is lighted loaded. So, Cell1 will suffer from high handover failure rate and high call blocking rates when UEs belonging to other cells handover into the cell1. However, the cell 2 is less heavy loaded and low radio resource utilization. Then MLB algorithms bias the handover region between neighboring cells and handover the UEs to the cell2. This initial scene has been shown, but another items need to be described further. 


\section{A. Handover Process}

In LTE system, handover can be triggered when a UE detects that a neighboring cell offer better received signal strength from the serving cell. We suppose that UEs handover from source cell1 to target cell2. The A3 event can be expressed as:

$$
M_{2}>M_{1}+\left(H M_{1}-C I O_{1,2}\right)
$$

where $M_{1}$ and $M_{2}$ denote the received signal strength UE from cell1 and from cell2, $H M_{1}$ the hysteresis parameter of cell1 for $\mathrm{A} 3$ event, and $\mathrm{CIO} \mathrm{O}_{1,2}$ the cell individual offset set by cell1 for cell2 and is the key parameter to be adjusted to make load balance between cells [4]. If $M_{1}$ and $M_{2}$ satisfy the A3 event by Eq.(1) during one TTT, then the handover from cell1 to cell 2 is triggered. Fig. 2 illustrates the handover process: The point A means that the entering condition for A3 event has just been satisfied, the point $B$ means that the UE starts to handover from cell1 to cell2, and the point $C$ means the that handover is just finished. The time period while the UE moves from point A to point B equals to TTT.

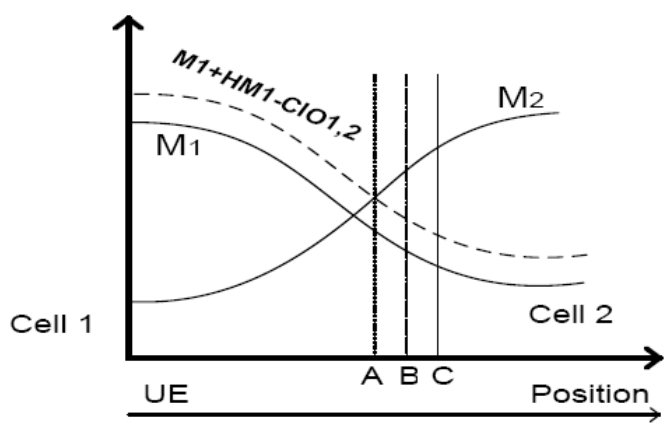

Fig. 2. A3 event in LTE.

\section{B. Resource Assignment}

In this paper, the focus is on the LTE downlink which is a localized OFDMA system. According to the LTE system, physical resource blocks (PRBs) are the smallest unit. When a UE becomes active, the system will assign resources to it. The resource assignment is just like the scheme in due to the main consideration on MLB algorithm. The number of PRBs assigned to the UE is briefly taken into account below [5].

Active UE has a bit rate requirement which is denoted as $D_{u}$ and the required number of PRBs to meet $D_{u}$ can be denoted as $\widehat{N}_{u}$. The required resources $\widehat{N}_{u}$ depends on the $S I N R_{u}$. Given the $S I N R_{u}$, we can get the data rate per PRB using a throughput mapping $R_{u}=R\left(S I N R_{u}\right)$. The most prominent example for such a function $R()$ is Shannon's equation. We can express the Shannon formula:

$$
\begin{gathered}
R_{u}=\log _{2}\left(1+\operatorname{SINR}_{u}\right) \\
\operatorname{SINR}_{u}=\frac{P_{c} \cdot L_{x(u)}}{N+\sum_{c \neq X_{(u)}} \rho_{c} \cdot P_{c} \cdot L_{c}}
\end{gathered}
$$

where $P_{c}$ is transmit power for a cell and $\mathrm{N}$ denotes as thermal noise. The $X_{(u)}, L_{x(u)}$ and $L_{c}$ denote the currently serving cell of UE $u$, the path loss with shadow fading from the currently serving cell and other interference cell, respectively. The $\rho_{c}$ denotes the load of the cell c.

With the definition, the amount of required PRBs $\hat{N}_{u}$ can be obtained as:

$$
\hat{N}_{u}=\frac{D_{u}}{R(\operatorname{SINR})}
$$

For reality, the actual amount of PRBs occupied by UE $u$ can be yielded:

$$
N_{u}= \begin{cases}\min \left(\hat{N}_{u}, K_{c}\right) & P R B_{\text {Left }}>\min \left(\hat{N}_{u}, K_{c}\right) \\ 0 & \text { else }\end{cases}
$$

where $K_{c}$ is a constant ( 2 in simulation) to keep PRBs occupied by the users with low channel quality under reasonable level. $P R B_{\text {Left }}$ denote the left PRBs of cells for accessing. From the equation (5), we can obtain that the users will be blocked when $N_{u}$ is zero $\left(P R B_{\text {Left }}<\min \left(\hat{N}_{u}, K_{c}\right)\right)$.

\section{Load Measurement}

Cell load is an important parameter which influences the MLB algorithm greatly. The MLB algorithm is carried out based on cell load measurement, so the performance lies on it heavily. We define cell load as the mean utilization of the total amount of physical resource blocks across the cell.

$$
\rho_{c}=\frac{\sum_{u \mid X(u)=c} N_{u}}{M_{P R B}}
$$

$M_{P R B}$ is the total number of resources in the cell. In this paper, we follow the real scenario and will not assign resources when there are not enough PRBs for new coming active UEs [6], [7].

\section{THE PROPOSED SCHEME}

\section{A. Adaptive Hysteresis Margin}

Usually the level of the hysteresis is constant. The adaptive hysteresis margin $(H M)$ is based on the modification of actual $H M$ value according to the position of the user in the cell. The $H M$ is decreasing with UE's moving closer to the cell border. The border of cells are neither regular circles nor hexagons since the system is not distance or signal level limited but it is interference limited. Therefore, the shape of the cells is strongly influenced also by the interference [8]. 
Hence, this paper further proposes to implement (Signal to interference plus noise ratio) SINR instead of (received signal strength indicator) RSSI for calculation of the actual level of $H M$. A signal level influenced by the interference and noise (IN) can be described according to the next equation:

$$
S I N R=T P_{s t}-P L-I N=R S S I-I N
$$

$T P_{s t}$ is transmission power of the station of interest. The SINR level is in different range of values than RSSI. Immediately it has to be related to the difference between maximum and minimum SINR in the observed area. Thus, the actual $H M$ level according to SINR is derived as follows:

$$
H M=\max \left\{H M_{\max } \times\left(1-10^{\frac{S I N N_{a c}-S I N R_{\min }}{S S I N R_{\max }}}\right)^{\text {exp }} ; H M_{\min }\right\}
$$

where $S I N R_{a c t}$ the actual $\operatorname{SINR}$ is measured by a UE; $S I N R_{\min }$ and $S I N R_{\max }$ are minimum and maximum values in the investigated area respectively, exp represents the exponent equal to 4 and $H M_{\text {min }}$ is the minimum $H M$ that can be set up equal to 0 [9].

The actual SINR of UE can be easily measured during UE's operation. It is usually performed with the purpose of handover decision and initiation. However, also the minimum and maximum SINR values also have to be known for the utilization of the adaptive $H M$. SINR $R_{\min }$ corresponds to the cell radius and to the SINR level, at which the UE is able to receive data. Therefore, it is set up as a fix value for each FAP and BS. The SINR $R_{\max }$ can be determined by two ways: 1) measurement of SINR by a FAP at the point of its location; or 2) monitoring and reporting of SINR by all UEs connected to the given FAP and than selecting the highest SINR from all known values as the $S I N R_{\max }$. The first way implies to equip all FAPs with ability to measure SINR. Hence it is not furthermore considered in the paper. The second approach utilizes the knowledge of previous SINR values in the area reported by UEs. Since the channel is time variant, the time interval for selection of $S I N R_{\max }$ should be determined.

\section{B. Load Balancing}

We assume that the serving cell named Cell1 is over loaded. It will choose the light loaded target cell named Cell2 to balance the load. To achieve this purpose, Cell1 should adjust the handover parameters to make the UEs handover from Cell1 to Cell2 more easily while make the UEs handover from Cell2 to Cell1 more difficultly. This is equivalent to that the handover position should be closer to Cell1 whether UEs handover from Cell1 to Cell2 or handover from Cell2 to Cell1. To make the handover position of UEs handover from Cell2 to Cell1 move from point A to point A1 (Fig. 3), the handover parameters are adjusted as follows:

$$
C I O_{2,1 \min }=M_{t h}+H M_{2}-M_{1 \max }
$$

$$
\begin{gathered}
\Delta_{1}=\left(C I O_{2,1}-C I O_{2,1 \mathrm{~min}}\right) \times\left(1-\frac{\operatorname{Load}_{2}}{\operatorname{Load}_{1}}\right) \\
C I O_{2,1}^{*}=C I O_{2,1}-\Delta_{1}
\end{gathered}
$$

where $M_{t h}$ is the threshold of the received signal strength required by handover, $M_{1 \max }$ the received signal strength of the UE from Cell1 when the received signal strength the UE receives from Cell2 is equal to $M_{t h}, \mathrm{CIO}_{2,1 \mathrm{~min}}$ a critical value that makes the $\mathrm{A} 3$ event just to be satisfied. In order to prevent the too late handover, when UE moves from Cell2 to Cell1, $\mathrm{CIO}_{2,1}$ should be adjusted to $\mathrm{CIO}_{2,1}^{*}$ which is between the value of $\mathrm{CIO}_{2,1}$ and $\mathrm{CIO}_{2,1 \mathrm{~min}}$. The step $\Delta_{1}$ is decided by the load of Cell1 and Cell2. The Smaller value of (Load2/Load1) and the larger the value is $\Delta_{1}$. Then closer point A1 to Cell1.

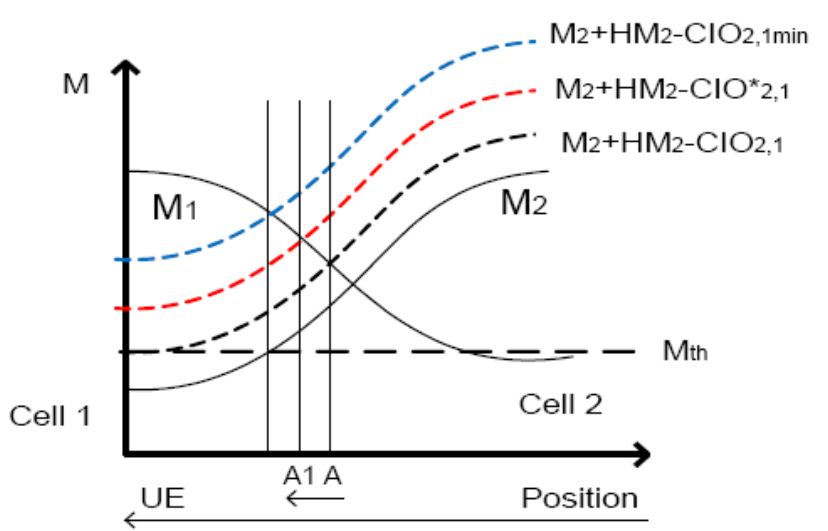

Fig. 3. Adjust point A to A1.

To make the UEs in Cell1 handover to Cell2 more difficult, we will make the UEs handover positions from Cell1 to Cell2 closer to Cell1. This means point B will be changed to point B1 as illustrated in Fig. 4. So the point A1 is the new handover position when UEs move from Cell2 to Cell1 and point B1 is the new handover position when UEs move from Cell1 to Cell2. In consideration of Ping-Pong handover, the point B1 should be further from Cell1 than the point A1, but closer to Cell1 than the point $\mathrm{B}$.

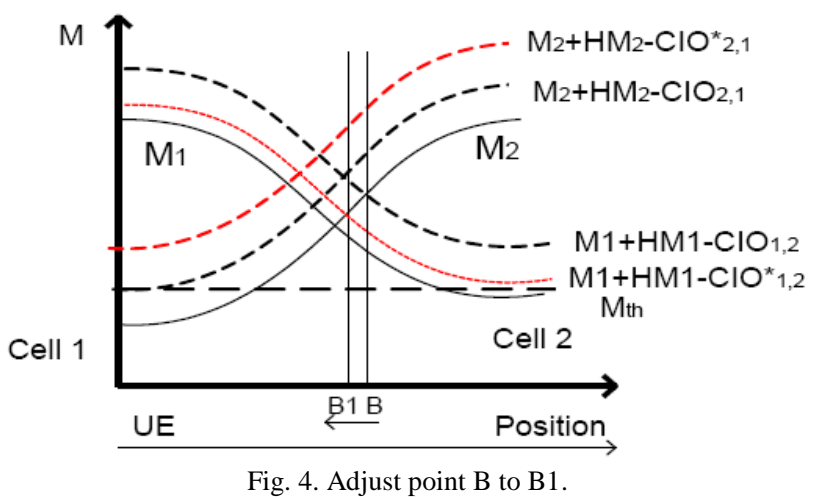

Optimization restriction: We can get the adjustment on $\mathrm{CIO}_{1,2}^{*}$ and $\mathrm{CIO}_{2,1}^{*}$ is restricted in the allowed range. 


$$
\left\{\begin{array}{c}
\mathrm{CIO}_{1,2}^{*}+\mathrm{CIO}_{2,1}^{*}<\mathrm{HM}_{1}+\mathrm{HM}_{2} \\
\mathrm{CIO}_{2,1 \mathrm{~min}}<\mathrm{CIO}_{2,1}^{*}<\mathrm{CIO}_{2,1} \\
\mathrm{CIO}_{1,2}^{*}>\mathrm{CIO}_{1,2}
\end{array}\right.
$$

This proposed scheme is triggered by MLB, so the MLB procedure should work as follows:

1) The MLB function in Cell1 collects the load statistics of neighbor cells.

2) The Cell1 detects an eligible neighbor (Cell2) and requests its handover parameters $\mathrm{CIO}_{2,1}$ and $\mathrm{H} 2$.

3) The Cell1 adjusts the parameter $\mathrm{CIO}_{1,2}$ and $\mathrm{CIO}_{2,1}$ to $\mathrm{CIO}_{1,2}^{*}$ and $\mathrm{CIO}_{2,1}^{*}$ according to equality and inequality.

4) The Cell1 transfers the parameter $\mathrm{CIO}_{2,1}^{*}$ to Cell2, and both of the Cell1 and Cell2 synchronize the new parameters.

\section{PERformance EVAluation}

We evaluate the proposed scheme by LTE system level simulator. LTE-Sim is an open source framework to simulate LTE networks mainly developed by G. Piro and F.Capozzi at "Politecnico di Bari"[10]. During the simulation time, UEs always connect to the network and keep calling. When the simulator starts running, every UE chooses random direction and moves straight with a specific speed. Compare between the original scheme and the proposed scheme, we can see in original scheme MLB operates without restriction, in the proposed scheme the operation of MLB is restricted in the allowed range to avoid the load problem. Other main simulation parameters summarized in Table I.

TABLE I: SIMULATION PARAMETER

\begin{tabular}{|c|c|}
\hline Parameter & Value \\
\hline System Bandwidth & $5 \mathrm{MHz}$ \\
\hline Frequency & $2 \mathrm{GHz}$ \\
\hline Radius of Microcell & $0.5 \mathrm{~km}$ \\
\hline Radius of Picocell & $0.05 \mathrm{~km}$ \\
\hline eNB Tx power & $46 \mathrm{dBm}$ \\
\hline Pico Tx power & $30 \mathrm{dBm}$ \\
\hline Thermals Noise & $-174 \mathrm{dBm} / \mathrm{Hz}$ \\
\hline Pathloss Model & As 3GPP $36.814 \mathrm{~V} 1.5 .2$ \\
\hline Load Threshold & 0.8 \\
\hline Cell layout & Wrap around, $7 \mathrm{Micro}$ \\
\hline Scheduler & PNodeBs \\
\hline Traffic Model & $\mathrm{CBR}$ \\
\hline UE speed & $30 \mathrm{~km} / \mathrm{h}, 120 \mathrm{~km} / \mathrm{h}$ \\
\hline
\end{tabular}

\section{A. Simulation Results}

We present the simulation result for performance comparison between the original scheme and proposed scheme. The MLB modifies the parameter CIO to a reasonable range. Fig. 5a), 5b) and 5c) show that the handover success rate, RLF rate and ping-pong handover are significantly better than the original scheme. Due to the fact that MLB function in the proposed scheme adjusts the parameter $\mathrm{CIO}$ to a reasonable value according to the load distribution of neighboring cells.

Fig. 5d) show that the system average throughput increase. This is because after the MLB operation, the handover positions of UEs moving from overloaded cell to its light load neighbor cell and the handover position of UEs moving from the light load neighbor cell to the overload cell all get closer to the overloaded cell. Therefore, the UEs can handover from the overloaded cell to its neighbor cell more easily, and the UEs handover from the neighbor cell to the overload cell more difficultly. This makes the UE growth rate of the overloaded cell is lower than the UE decrement rate. So the load level of the overload cell decreases.

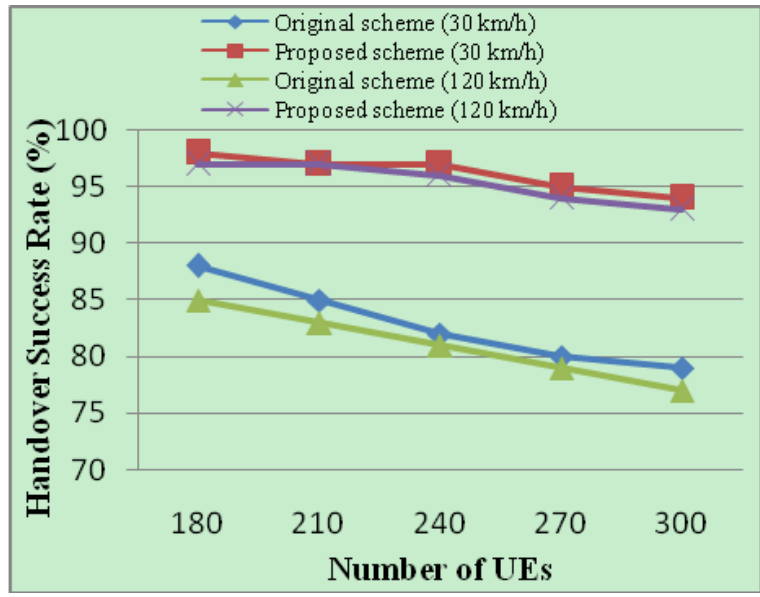

Fig. 5a). Handover success rate.

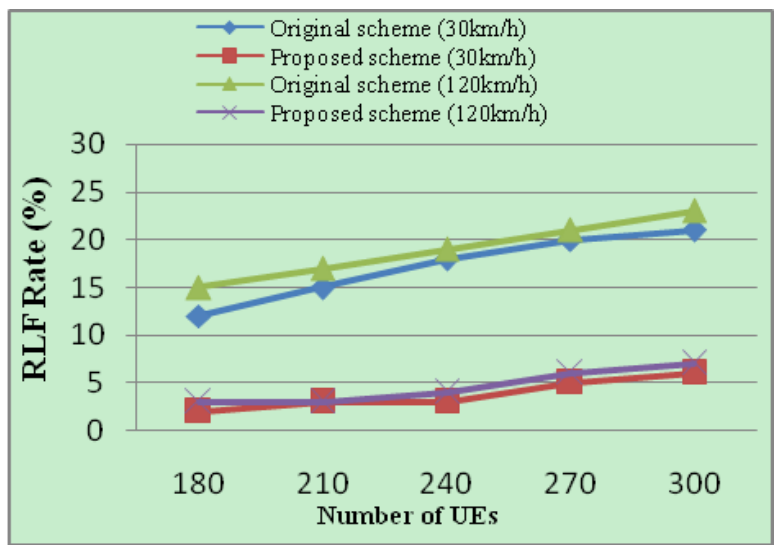

Fig. 5b). Radio link failure rate.

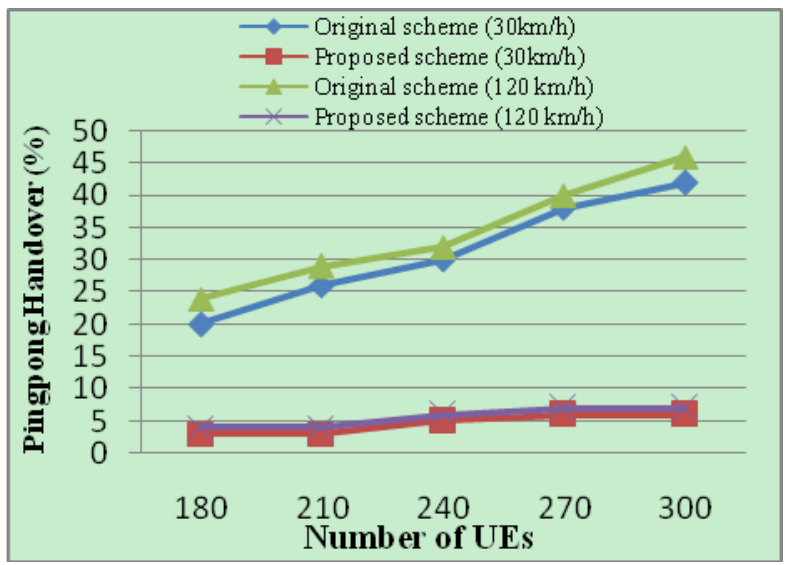

Fig. 5c). Ping-pong handover rate. 


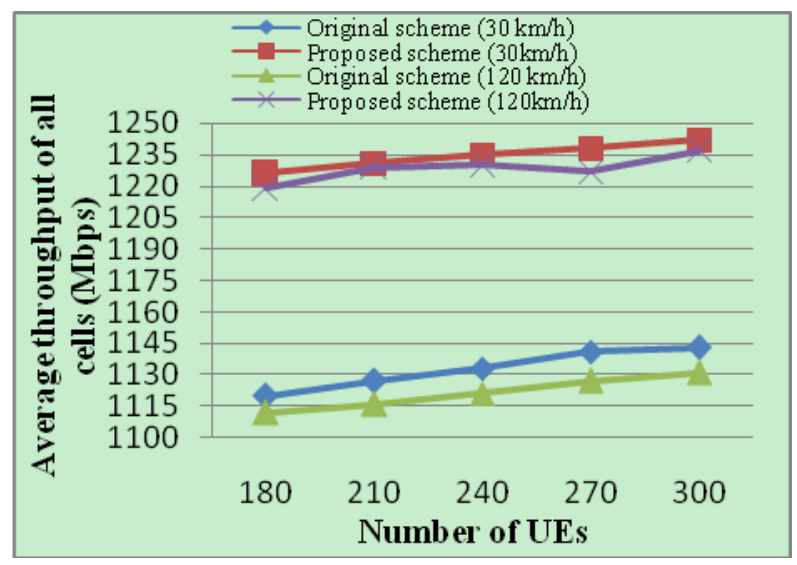

Fig. 5d). Average throughput.

\section{CONCLUSIONS}

In this paper, MLB methods have been proposed for load balancing in LTE HetNets scenario. The proposed scheme is based on the load distribution of neighboring cells to adjust the handover parameters. System level simulations have shown that the proposed schemes can greatly increase the handover success rate, the RLF rate, ping-pong handover rate and throughput. Furthermore, it can reduce the number of overloaded cell and improve the overall system throughput.

\section{REFERENCES}

[1] S. Zaidi and G. Talagery, "Developing and integrating a high performance HET-NET," 4G Americas, October 2012.

[2] N. Saquib, E. Hossain, and D. I. Kim, "Fractional frequency reuse for interference management in LTE-advance HetNets," IEEE Wireless Communications, pp. 113-122, April 2013.

[3] M. Ravichandra et al., "A survey on handovers literature for next generation wireless networks," IJARCCE, vol. 2, issue 12, December 2013.
[4] 3GPP standardization, "Evolved universal terrestrial radio access (E-UTRA); Radio resource control (RRC)," Release 8, V8.5.0, TS $36.331,2009$

[5] 3GPP standardization, "Evolved universal terrestrial radio access (E-UTRA); Physical layer procedures," Release 10, V10.2.0, TS 36.213, 2011.

[6] P. Munoz, R. Barco, and I. Bandera, "On the potential of handover parameter optimization for self-organizing networks," IEEE, vol. 62, no. 5, pp. 1895-1905, June 2013.

[7] 3GPP standardization, "Evolved universal terrestrial radio access (E-UTRA); Layer 2 - Measurements," Release 10, V10.2.0, TS 36.314, 2011.

[8] Z.-Q. Liu, P.-L. Hong et al., "Conflict avoidance between mobility robustness optimization and mobility load balancing," IEEE, 2010.

[9] Z. Becvar and P. Mach, "Adaptive hysteresis margin for handover in femtocell networks," FP7 project FREEDOM IST-248891 STP.

[10] G. Piro, L. A. Grieco, G. Boggia, F. Capozzi, and P. Camarda, "Simulating LTE cellular systems: An open source framework," IEEE Trans. Veh. Technol., Oct. 2010.

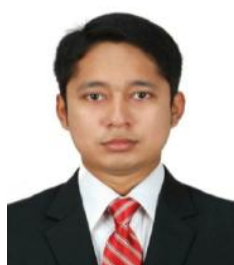

Ranada Prasad Ray received his B.Sc. degree in electronics and telecommunication engineering from Daffodil International University, Bangladesh, in 2010. Currently he is a M.Sc. student of the School of Communication and Information Engineering at Chongqing University of Posts and Telecommunications, China. His research interests include the load balancing in heterogeneous networks, handover decision for mobility management and quality of service in wireless networks.

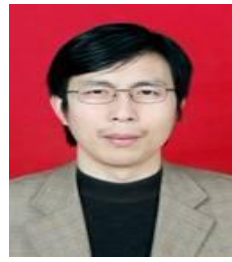

Lun Tang received the Ph.D. degree in the Chong Qing University, China in 2010. Since 2004, he has been with the College of Communication Engineering at the Chong Qing University of Posts and Telecommunications, China. He is currently a professor. In the past few years he has focused mainly on wireless networks (cellular networks, vehicle ad hoc networks). 